\title{
Pharmacogenomics and Adverse Drug Reactions: Carbamazepine Induced Steven Johnsons Syndrome
}

\author{
Akhila Dadwai*, Karthika Navar, Manasa Bagilipeddola, Rajareddy Rajannagari, \\ Satyavardhan Rao Nittu
}

Arya College of Pharmacy, Osmania University, Kandi, Sangareddy, Telangana, INDIA.

\begin{abstract}
Background: Carbamazepine, an anticonvulsant and a mood-stabilizing drug is the main cause of the StevensJohnson Syndrome (SJS) and its related disease, toxic epidermal necrolysis (TEN), in Southeast. Asian countries. Carbamazepine-induced SJS-TEN is strongly associated with the HLA-B*1502 allele. Case report: An eight-yearold female was admitted to the pediatric ward with chief complaints of macular rash all over the body, itching and giddiness for a week, on the evaluation of her history reveals that she is an epileptic on medication Carbamazepine $100 \mathrm{mg}$ once-daily dose. On the $10^{\text {th }}$ day of CBZ therapy, the patient developed macular rashes all over the body and CBZ was discontinued. Hence, the final diagnosis was made as Carbamazepine induced Steven-Johnson syndrome.
\end{abstract}

Key words: Carbamazepine, Steven-Johnson syndrome, Toxic epidermal necrolysis, HLA-B*, Adverse reactions, Pharmacogenomics.

\section{INTRODUCTION}

Carbamazepine (CBZ) is a derivative of iminostilbene with a carbamyl group at the 5 position, which is meant for its anti-seizure activity. It is used as a first-line antiepileptic drug in partial and tonic-clonic seizures. In addition, it is also used as a choice of drug in trigeminal neuralgia. Its structure is similar to tricyclic antidepressants; it is a preferred choice of drug in depression and maniac patients due to its moodstabilizing effects. The most commonly observed antiepileptic drugs (AEDs) induced cutaneous adverse drug reactions (cADRs) include maculopapular exanthema (MPE), Stevens-Johnson Syndrome (SJS), Toxic Epidermal Necrolysis (TEN) and Drug Reaction with Eosinophilia and Systemic Symptoms (DRESS). ${ }^{1}$ There is an increased risk for Stevens-Johnson syndrome/toxic epidermal necrolysis (SJS/TEN) among HLA-B*15:02 positive patients and CBZ induced reactions such as Hypersensitivity Syndrome (HSS) whereas MPE occurs in Human Leukocyte antigen (HLA-A*31:01) positive patients and SJS/TEN and acute generalized exanthemata's pustulosis (AGEP) rarely occur. ${ }^{2}$
Recently, many studies have shown that carbamazepine-induced hypersensitivity reactions are associated with mutations in human leukocyte antigen (HLA) genes. Supportively, they are strong phenotype and ethnicity-specific. HLA-B*15:02 (first reported in 2004) and HLA-A*31:01 (first reported in 2006) are the two major genetic risk alleles that are identified for CBZinduced hypersensitivity reactions. Data from the Han Chinese group supports the hypothesis that genetic susceptibility to CBZ-induced SJS/TEN might be ethnicityspecific. A strong association between HLA-B*1502 and AED-induced Subcutaneous Reactions was found. Most of the HLA-B*15:02 positive patients are, to a greater extent, reported from Asian countries, including China, Thailand, Malaysia and India, whereas it is rare among Caucasians or Japanese. HLA-B*31:01 has been detected in Caucasian, Japanese, Korean, Chinese and patients of mixed origin; however, HLA-A*31:01 is also common in most ethnic groups. ${ }^{3}$

US FDA recommendations for Genetic
DOI: 10.5530/ijopp.14.2.27

Address for correspondence: Dr. Akhila Dadwai Pharm.D Arya College of Pharmacy, Kandi, Sangareddy-502285, Telangana, INDIA.

Phone no: +91-9705723433 Email Id: akhiladadvai@gmail. com

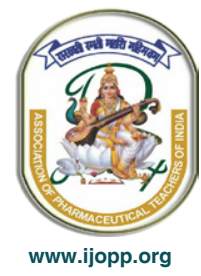


testing of these haplotypes have been recently updated to ensure safe carbamazepine therapy. ${ }^{4}$ HLA-B* 15:02 allele genotyping is suggested for all CBZ-naive patients originating from populations where this allele is common and whose origin is unknown, but it is optional in patients originating from populations where this allele is rare, before initiation of CBZ therapy. Besides, HLA-A*31:01 allele is usually observed in most races. ${ }^{5}$ Thus, genotyping for this allele is an important recommendation for all CBZ-naive patients before starting CBZ therapy. ${ }^{6}$

\section{CASE REPORT}

An 8-year-old female was admitted to the pediatric ward of District Government Hospital Sangareddy, with chief complaints of macular rash all over the body, itching and giddiness for a week. History of her present condition states that the patient had a fever and vomiting one week ago. Upon general examination, her vitals were: Temperature $101 \mathrm{~F}$, Pulse rate $90 / \mathrm{min}$, Blood pressure $110 / 70 \mathrm{mmHg}$ and Respiratory rate $30 / \mathrm{min}$. Laboratory investigations revealed elevated levels of Erythrocyte Sedimentation Rate $(100 \mathrm{~mm} / \mathrm{hr}$.) shows the inflammatory conditions. On the evaluation of her history reveals that she has epilepsy on medication CBZ $100 \mathrm{mg}$ once-daily dose. On the $10^{\text {th }}$ day of CBZ therapy, the patient developed macular rashes all over the body and CBZ was discontinued. Hence, the final diagnosis was made as Carbamazepine induced Steven-Johnson syndrome.

The patient was treated with parenteral Hydrocortisone and ceftriaxone, topical Betamethasone, Calamine topical lotion, along with IV fluids and other supportive measures. The patient condition improved and was discharged after three days of hospital stay. The patient's attenders were counseled regarding the condition of the patient and were discharged with Tab Prednisolone $20 \mathrm{mg}$ once daily, Tab Pantoprazole, Cap. Methylcobalamin, Tab. Valproate once daily. A causality analysis was done using Naranjo's algorithm, WHO-UMC Scale and the present ADR was found to be 'PROBABLE' with Carbamazepine. The reaction was "PROBABLY PREVENTABLE," according to the modified Schumock and Thornton scale and the modified Hart wig and Siegel severity scale showed that the reaction is SEVERE (Level 6).

\section{Patient Alert Card}

Alert card was issued to the patient attenders and was advised to reveal this medication allergy to the other physicians before getting a prescription from them to avoid this drug, which causes SJS or TEN.

\section{Patient Ascent Form}

The Case was clerked from the District Government Hospital Sangareddy, Telangana. The written informed Ascent form was taken from the patient attenders for the presentation/publication of the case report.

\section{DISCUSSION}

There are random reports of Steven-Johnson Syndrome with the use of CBZ. Approximately three persons per million per week may experience life-threatening dermatological syndromes with the use of $\mathrm{CBZ}^{7}$ In this case, the patient developed itchy erythematous lesions and macular rashes. Current evidence indicates that Carbamazepine induced SJS/TEN is a predictable, specific, delayed hypersensitivity immune reaction involving human leukocyte antigen (HLA) alleles specific for Carbamazepine and other drugs in defined populations. HLA-B*15:02 and HLA-B*31:01 have been associated with carbamazepine-induced SJS in Asian (Han Chinese, Thai, Indian and Malaysian) and Caucasian populations, respectively. ${ }^{7,8}$ In a study conducted by the Han Chinese group, it was found that $\mathrm{B}^{*} 1502, \mathrm{Cw}^{*} 0801$, A*1101 and DRB1*1202 in the HLA region were reported at a higher frequency in $\mathrm{CBZ}-\mathrm{SJS}$ patients than the controls. Interestingly, HLA-B*1502 was present in $100 \%$ of CBZ-SJS patients making it a critical checkpoint before initiating CBZ therapy. ${ }^{9}$ Supportively, susceptibility to HLA induced cutaneous reactions is phenotypespecific. Current data also support the hypothesis that genetic susceptibility to CBZ-induced SJS/TEN might be ethnicity specific.

PharmGKB (The Pharmacogenetics Knowledge Base) and CPIC (The Clinical Pharmacogenetics Implementation Consortium) strongly recommends not to use CBZ if alternatives are available when the patient is CBZ-naive. If the patient has previously used Carbamazepine for longer than three months without the incidence of cutaneous reactions, $\mathrm{CBZ}$ can be considered as a choice with cautious monitoring.

\section{CONCLUSION}

Adverse drug reactions, especially in the pediatric population, is an important concern. So, rigid ADR monitoring among pediatric patients is necessary to ensure the safety of drug therapy. Patient Care should be taken while prescribing the drug, as Carbamazepine is well reported in the literature for causing SJS the underlying cause might be the genetic variant in HLA and should be counseled about the possible side effects. Therefore, Carbamazepine must not be challenged to avoid further fatal reactions. Early diagnosis helps 
to prevent secondary infections and subsequent complications. Pharmacovigilance, which deals with the identification, assessment and prevention of ADRs, can help in providing continuous information on medication safety and appropriate use. Preemptive and reactive Pharmacogenomics testing makes more effective and fewer side-effects.

\section{ACKNOWLEDGEMENT}

We want to express our sincere appreciation to all those who provided us the possibility to complete this report. This report would not have been possible without the supervision of our professor Dr. Rajareddy whose contribution to stimulating suggestions and encouragement helped us to coordinate, especially in writing this report.

\section{CONFLICT OF INTEREST}

The authors declare no conflict of interest.

\section{ABBREVIATIONS}

TEN: Toxic Epidermal necrolysis; WHO: World Health Organization; HLA: Human Leukocyte Antigen; SJS: Steven-Johnsons syndrome; CBZ: Carbamazepine; AED: Anti-epileptic drugs; MPE: Maculopapular exanthema.

\section{REFERENCES}

1. Fang PY, Ying Y, Qinyuan Z, Sheng-an C, Xiaodan F, Sijia Y, et al. Research on Susceptible Genes and Immunological Pathogenesis of Cutaneous Adverse Drug Reactions in Chinese Hans. In: Journal of Investigative Dermatology Symposium Proceedings. 2015;7(1):29-31.

2. Lee JW, Aminkeng F, Bhavasar APK, Shaw BC, Carleton MR. The emerging era of pharmacogenomics: Current successes, future potential and challenges. In Clin Genet. 2014;86(1):21-8.

3. Shuen-lu H, Wen-Hung C, Shiou-Hwa J, Wen-Chieh C, Yun-Ting C, Woan-Ruoh L, et al. Genetic susceptibility to carbamazepine-induced cutaneous adverse drug reactions. In Pharmacogenetics and Genomics. 2006;16(4):297-306.

4. Novartis. Carbamazepine (Tegretol) [package insert]. U.S. Food and Drug Administration website https://www.accessdata.fda.gov/drugsatfda_docs/ label/2007/016608s098lbl.pdf.

5. Caroline FT, Teri EK, Russ BA. PharmGKB: The pharmacogenetics and pharmacogenomics knowledge. The Pharmacogenomics Journal. 2004;4(1):1.

6. Elizabeth JP, et al. Clinical Pharmacogenetics Implementation Consortium (CPIC) Guideline for HLA Genotype and use of Carbamazepine and Oxcarbazepine. 2017.

7. Sukasem C, Chaichan C, Nakkrut T, Satapornpong P, Jaruthamsophon $\mathrm{K}$, et al. Association between HLA-B Alleles and Carbamazepine-Induced Maculopapular Exanthema and Severe Cutaneous Reactions in Thai. In Journal of Immunology Research. 2018.

8. Amstutz U, Shear NH, Rieder MJ, Hwang S, Fung V, Nakamura $\mathrm{H}$, et al. Recommendations for HLA-B*15:02 and HLA-A*31:01 genetic testing to reduce the risk of carbamazepine-induced hypersensitivity reactions. In Epilepsia. 2014;55(4);496-506.

9. Wen-Hung $\mathrm{C}$, Shuen-lu $\mathrm{H}$, Hong-Shang $\mathrm{H}$, Mo-Song $\mathrm{H}$, Li-Cheng $\mathrm{Y}$, Hsin-Chun $\mathrm{H}$, et al. Medical genetics: A marker for Stevens-Johnson syndrome. In Nature. 2004;428(6982):486. 